\title{
La participation sur les espaces de contenus générés par les utilisateurs, une exploitation collaborative
}

Marc-Olivier GOYETTE-CÔTÉ and Philippe LANGLOIS

\section{(2) OpenEdition \\ Journals}

Electronic version

URL: http://journals.openedition.org/ticetsociete/1407

DOI: 10.4000/ticetsociete. 1407

Publisher

Association ARTIC

Electronic reference

Marc-Olivier GOYETTE-CÔTÉ and Philippe LANGLOIS, « La participation sur les espaces de contenus générés par les utilisateurs, une exploitation collaborative », tic\&société [Online], Vol. 8, N 1-2 | 1er semestre 2014 et 2ème semestre 2014, Online since 31 May 2014, connection on 30 April 2019. URL : http://journals.openedition.org/ticetsociete/1407 ; DOI : 10.4000/ticetsociete.1407 
tic\&société - 8 (1-2), 2014

La participation sur les espaces de contenus générés par les utilisateurs, une exploitation collaborative

Marc-Olivier GOYETTE-CÔTÉ

Faculté de communication

CRICIS

UQAM

goyette.marco@gmail.com

Philippe LANGLOIS

Département de sociologie

UQAM

langl.philippe@gmail.com 
La participation sur les espaces de contenus générés

par les utilisateurs, une exploitation collaborative

\section{La participation sur les espaces de contenus générés par les utilisateurs, une exploitation collaborative}

Marc-Olivier GOYETTE-CÔTÉ est doctorant en cotutelle en communication à I'Université du Québec à Montréal (UQAM) et en sciences de l'information et de la communication à l'Université Sorbonne-Nouvelle Paris III. II est également adjoint de recherche au Centre de recherche interuniversitaire sur la communication, l'information et la société (CRICIS). Son doctorat porte sur les effets structurants des infomédiaires dans les industries de la culture et les industries de la communication. II a collaboré à plusieurs projets de recherche sur les liens entre concentration, convergence et pluralisme de l'information et publié plusieurs articles sur l'économie politique du journalisme québécois dans des revues internationales. Ses recherches actuelles portent sur le journalisme, les données personnelles et les enjeux de l'intermédiation des contenus.

Philippe LANGLOIS est doctorant en sociologie à l'Université du Québec à Montréal (UQAM) après avoir obtenu une maîtrise en science politique. Ses recherches portent sur les interventions des banques centrales lors de la crise financière de 2008-2009 et leurs répercussions sur le système financier et monétaire. II a par ailleurs travaillé en collaboration avec plusieurs groupes de recherche en économie politique comme le CEIM (Centre d'étude sur l'intégration et la mondialisation), l'IRIS (Institut de recherche et d'informations socio-économiques) et I'IREC (Institut de recherche en économie contemporaine) sur des sujets variés comme les PPP dans le domaine de la santé ou le financement des organismes communautaires.

Résumé : En partant des notions de prosumer et de crowdsourcing, nous montrons en quoi la théorie critique permet de saisir les mutations des formes de participation en ligne. Nous argumentons que le prosuming s'inscrit dans une logique de récupération capitalistique de la participation dans une logique de délocalisation du travail en dehors des murs des entreprises. Ce texte construit sur un débat sur les catégories de l'analyse marxiste traditionnelle que sont le temps de travail et le travail salarié (Fuchs, 2010, 2012 ; Arvidsson et Colleoni, 2012). Nous cherchons à montrer que l'insertion des formes de prosuming dans les stratégies commerciales des entreprises procède d'un processus de liquéfaction des formes du travail dans le capitalisme avancé et doit donc être analysé comme une forme de travail. 


\section{Marc-Olivier GOYETTE-COTE et Philippe LANGLOIS}

Mots-clés : prosumer, crowdsourcing, capitalisme avancé, sociologie du travail, théorie critique

Abstract : Building on the notions of prosumer and crowdsourcing, this article shows how critical theory allows to grasp the mutations of participatives forms online. We argue that prosuming proceeds from a capitalistic recuperation of participation in a logic of delocalization of work outside the web enterprise's wall. This article builts on a debate on the traditional marxist categories of labor-time and wage-labor (Fuchs, 2010, 2012 ; Arvidsson et Colleoni, 2012). We aim to show that the insertion of the different forms of prosuming in commercial strategies proceeds for a mouvement of liquefaction of labor forms in the context of advanced capitalism, and hence, should be analazed as labor.

Keywords : prosumer, crowdsourcing, advanced capitalism, work sociology, critical theory

Resumen: Usando los conceptos de prosumer y crowdsourcing, argumentamos cómo la teoría crítica captura mutaciones en formas de participación en línea. Sostenemos que el prosuming es parte de una lógica de recuperación del capital de la participación en una lógica de deslocalización de trabajo a fuera de los muros de las empresas. Este texto se basa sobre un debate de las categorías de análisis marxista tradicional que son el tiempo de trabajo y el trabajo asalariado (Fuchs, 2010, 2012 ; Arvidsson y Colleoni, 2012). Buscamos demostrar que la inclusión de las formas de prosuming en las estrategias de negocio se debe a un proceso de licuefacción de las formas de trabajo en el capitalismo avanzado y debe ser analizada como una forma de trabajo.

Palabras clave : Prosumer, crowdsourcing, capitalismo avanzado, sociología del trabajo, teoría crítica

\section{Introduction}

Dans le contexte du développement du web comme plateforme de production/diffusion/consommation de la culture, nous avons vu réapparaître les discours techno-déterministes sur la "société de l'information » tant du côté des chercheurs (Castells, 1997; Jenkins, 2004) que des professionnels (O'Reilly, 2006) ou des journalistes. Selon ces discours, l'internet aurait permis le grand bond en avant vers la "société de l'information", dans laquelle le travail 


\section{La participation sur les espaces de contenus générés par les utilisateurs, une exploitation collaborative}

manuel ne constituerait plus le cœur de l'activité économique. Dans cette soidisant "société de l'information", concrétisation partielle de la "société des loisirs » (Dumazedier, 1962), le prosumer, figure d'un producteur qui consomme son travail proposée par Toffler (1981), serait au centre du processus économique où le travail industriel serait désormais périphérique à la création de valeur et où la production devrait être perçue comme une forme d'engagement plutôt que comme une nouvelle forme de travail (Arvidsson et Colleoni, 2012).

La perspective critique nous amène toutefois à nous interroger sur le sens de l'activité des prosumers et à remettre en question les conditions de leur engagement. Plusieurs chercheurs critiques ont questionné les discours sur la "société de l'information » (George et Granjon, 2008). Certains ont souligné la marchandisation des espaces de contenus générés par les utilisateurs et la nature de l'activité des internautes (Comor, 2012). Suivant cette approche, nous avons souhaité dans cet article poser la question des modalités de récupération du travail des prosumers en cherchant à identifier si nous pouvions utiliser les catégories traditionnelles de l'analyse marxiste, notamment la théorie de la valeur-travail, pour saisir le cadre de leur activité dans les mutations en cours. En effet, pour certains auteurs dont Arvidsson et Colleoni (2012) la théorie du travail chez Marx, bien qu'expliquant en partie ces phénomènes, ne s'applique pas au phénomène du prosuming. De notre côté, nous argumentons avec Fuchs $(2010,2012)$ et Comor (2012) que l'activité des prosumers peut être analysée sous l'angle du travail, mais que nous devons pour cela mettre à jour certains aspects de la théorie marxiste traditionnelle, notamment la question du travail salarié et du quantum de temps de travail.

Dans cet article, nous défendrons donc le point de vue selon lequel le prosuming doit être analysé comme une nouvelle forme d'organisation du travail dans un continuum de liquéfaction des formes du travail. Pour ce faire, nous reviendrons dans un premier temps sur la constitution théorique de la figure du prosumer (Toffler, 1981) dans le contexte du développement du web comme nouveau lieu de création de valeur. Nous nous arrêterons ensuite sur la notion de crowdsourcing, afin de voir comment le prosumer est intégré aux logiques gestionnaires, ce qui implique que l'usufruit de son travail est accaparé de manière orientée par les propriétaires des plateformes de production/diffusion. Par la suite, nous examinerons le débat qui a lieu autour de l'opérationnalisation des concepts marxiens de valeur et de travail salarié dans le contexte du développement des espaces de contenus générés par les utilisateurs. Nous tenterons par la suite d'illustrer notre propos en étudiant la participation des internautes sur les plateformes Facebook, Amazon et Innoncentive. Nous 


\section{Marc-Olivier GOYETTE-COTE et Philippe LANGLOIS}

terminerons cet article en proposant un continuum des formes du travail qui permet de saisir la nature de l'activité des prosumers en ligne.

\section{De la notion de prosuming}

La notion de prosumer a été utilisée pour la première fois par Alvin Toffler dans son livre The Third Wave (1981). Selon lui, la séparation entre production et consommation est une invention de la société industrielle, "a wedge in society, that separated these two functions, thereby giving birth to what we now call producers and consumers ${ }^{1}(1981$, p. 266). Toffler affirme que dans les sociétés pré-industrielles, la production et la consommation étaient intimement reliées, puisque les personnes consommaient ce qu'elles produisaient. Ritzer et Jurgensen (2010) argumentent qu'en fait les consommateurs ont toujours eu un pied des deux côtés. Selon eux, même lors du pic de la révolution industrielle, la consommation et la production n'étaient jamais complètement séparées; les producteurs consommaient des matières premières et les consommateurs produisaient leurs repas.

Suite aux travaux de Toffler, Ritzer a approfondi la notion de prosumer en parlant du phénomène de McDonalization (1983) de la société. Selon lui, l'industrie de la restauration rapide aux États-Unis a commencé dès les années 1950 à insérer le consommateur dans la chaîne de travail. Pour Ritzer, les consommateurs deviennent en quelque sorte les serveurs (en transportant la nourriture et en ramassant leurs déchets) et les aides-cuisiniers de leurs repas (en y ajoutant les condiments et certains ingrédients ou en composant leurs salades dans les comptoirs à salades). En participant à leur expérience de repas au restaurant, les consommateurs auraient l'impression que le produit correspond davantage à leurs goûts et seraient contents de ne pas avoir eu à attendre d'être servis. Du côté des entreprises, il s'agit d'un gain en productivité, puisque le consommateur effectue des tâches qui incombaient auparavant à des employés salariés (Goyette-Côté, 2013). Ainsi, le prosumer serait le résultat de cette McDonaldisation, soit la "rationalization of service routines whereby consumers are expected to perform certain essential steps ${ }^{2}$ (Kleeman, et al., 2008, p. 7). Nous retrouvons cette même logique dans la stratégie d'IKÉA dont le modèle repose sur le fait d'éliminer les coûts d'assemblage et d'utiliser cette baisse des coûts de production en offrant ses produits à des prix plus bas que

\footnotetext{
1 «Une rupture dans la société, qui sépare ces deux fonctions, donnant ainsi naissance à ce que nous nommons maintenant producteurs et consommateurs » (traduction libre).

2 "Rationalisation des routines de service, où l'on s'attend à ce que les consommateurs exécutent certaines étapes essentielles du processus de production » (traduction libre).
} 
La participation sur les espaces de contenus générés

par les utilisateurs, une exploitation collaborative

ceux des meubles comprenant le salaire des employés en charge de l'assemblage. Ce qui s'apparente en une récupération marchande du principe alternatif du Do it yourself (fait le toi-même).

Dans le contexte de l'internet, le travail gratuit n'est pas un phénomène nouveau. En effet, il existe une tradition de travail gratuit et collaboratif entre informaticiens et ingénieurs dès les premiers systèmes de réseautage des ordinateurs dans les années 1960 (Proulx et Goldenberg, 2010). Abbate (1999) souligne à ce sujet que bien souvent les personnes ayant contribué au développement de l'internet étaient aussi ses premiers utilisateurs.

Cette tradition a contribué au développement de l'activité d'une certaine frange des utilisateurs sur le Web et a marqué l'imaginaire du web en le présentant comme une technologie émancipatrice. Cette vision a été propagée par les communautés du logiciel libre et par les premiers théoriciens de l'Internet (Ibid.). Comme le soulignent Proulx et Goldenberg, "plusieurs auteurs et artistes voyaient ainsi dans ces "communautés virtuelles", un espace collectif permettant d'expérimenter de nouveaux rapports d'échange caractéristiques d'une nouvelle société » (2010, p. 2). Ce mouvement, qui prend conscience de lui-même avec la "Déclaration d'indépendance du cyberespace " (PerryBarlow, 1996) propose une nouvelle forme d'organisation du travail plus horizontale présentée comme démocratique ou du moins, méritocratique (King et al., 1996). C'est cette vision d'une technologie émancipatrice qui a accompagné le développement du logiciel libre et influencé ces formes de travail. Toutefois, il importe de souligner que logiciel libre et travail gratuit ne sont pas obligatoirement liés, tout comme logiciel libre et capitalisme ne s'opposent pas toujours comme en fait foi l'importance du système d'exploitation Linux dans le marché des serveurs commerciaux.

Ce détour par le logiciel libre nous apparait essentiel puisqu'il symbolise les changements dans une forme de travail qui n'est pas généralisée sur le web, mais qui est mobilisée pour justifier le caractère non-aliénant des plateformes de contenus générés par les utilisateurs. En effet, nous observons un glissement dans les discours universitaires et professionnels qui associent des pratiques sommes toutes marginales aux pratiques de participation telles qu'elles se présentent dans la vaste majorité des espaces de contenus générés par les utilisateurs. C'est pourquoi, dans le cadre du présent article, nous nous attarderons principalement aux formes que prend la participation des utilisateurs dans un contexte de récupération marchande de la force de travail et du temps de loisir. 


\section{Marc-Olivier GOYETTE-COTE et Philippe LANGLOIS}

\section{L'interrelation entre crowdsourcing et prosumer}

Dans un article de la revue Wired (2006), Jeff Howe propose la notion de crowdsourcing, qu'il définit comme l'externalisation du travail vers le public. Dans cet article, il souligne que le crowdsourcing se définirait par: "The new pool of cheap labour: everyday people using their spare [ressources] to create content, solve problems, even do corporate $R$ et $D »^{3}(2006, p$. 1$)$. Howe veut démontrer tout au long de son article que de plus en plus d'entreprises font appel à des personnes en dehors de leurs listes de paie pour qu'elles effectuent des tâches qui autrement seraient effectuées par des employés salariés. Le terme crowdsourcing tire son origine des mots anglais crowd (foule) et outsourcing (délocalisation (offshore)). II s'agit donc de sortir des frontières nationales (offshoring) ou de l'entreprise (outsourcing) en faisant appel, soit à des entreprises tierces, soit à la population (crowdsourcing). Ainsi, les consommateurs en participant au processus de production peuvent apporter une valeur ajoutée, car ils possèdent des capacités spécifiques qui sont des ressources économiquement viables (connaissances techniques ou sociales, temps libre à disposition, etc.) pour les entreprises. Par ailleurs, les prosumers sont systématiquement intégrés dans les structures corporatives "where their actions can be monitored and manipulated by corporate managers much as if they were employees " ${ }^{4}$ (Kleeman et al., 2008, p. 7). Dans une recherche publiée en 2008, Kleeman, Voß et Rieder ont identifié sept modalités de crowdsourcing, soit la participation du consommateur dans: 1) le développement et la configuration des produits ; 2) dans la conception des produits; 3) dans les "Competitive bids on specifically defined tasks or problems" (Kleeman et al., 2008, p. 14); 4) dans des lignes ouvertes permanentes ; 5) dans les reportages communautaires ; 6) dans la notation des produits et profilage des consommateurs et; 7) dans le support " consommateurs - consommateurs » (ibid.). II appert que le phénomène du crowdsourcing est protéiforme et recouvre des pratiques extrêmement variées nécessitant aussi bien des bagages techniques importants que le simple fait de cocher une case d'un sondage (Goyette-Côté, 2013). II nous apparaît que nous devons analyser l'activité des prosumers dans le cadre d'une organisation de travail que nous pouvons qualifier de crowdsourcing.

\footnotetext{
3 "Le nouveau bassin de main-d'oeuvre à bon marché: des citoyens ordinaires qui utilisent leurs ressources et leurs habiletés pour créer du contenu, résoudre des problèmes et même faire de la recherche et du développement " (traduction libre).

${ }^{4}$ "Où leurs actions peuvent être surveillées et manipulées par les chefs d'entreprise comme s'ils étaient des employés " (traduction libre).
} 
La participation sur les espaces de contenus générés par les utilisateurs, une exploitation collaborative

Les différentes manifestations du crowdsourcing et la notion de prosumer illustrent clairement le fait que l'intervention de l'individu-consommateur est la pièce maîtresse du processus de valorisation des firmes ayant entrepris des activités «participatives» sur l'internet. II en ressort que l'activité des internautes présente deux facettes : d'un côté, leur participation de plus en plus forte à la production de contenus sur Internet fait qu'ils en retirent des satisfactions (Proulx et al., 2011), et de l'autre, les entreprises qui instrumentalisent cette participation croissante pour rentabiliser leurs activités. Voilà donc comment les prosumers sont intégrés dans les stratégies des entreprises qui possèdent les espaces de contenus générés par les utilisateurs. Nous nous arrêterons maintenant sur les notions de travail salarié, de temps de travail et de valeur dans le contexte du développement des espaces de contenus générés par les utilisateurs.

\section{La création de valeur dans le contexte des UGC : exploitation ou engagement ?}

Les plateformes associées au «web 2.0 » représentent selon Fuchs (2010) des lieux privilégiés pour l'exploitation du travail non rémunéré. En effet, ces plateformes sont orientées de manière à externaliser une partie plus ou moins importante de la production vers les internautes. Comme le souligne Fuchs, "one accumulation strategy is to give them free access to services and plateforms, let them produce content, and to accumulate a large number of producers that are sold as a commodity to advertisers $»^{5}(2010$, p. 191). II s'agit d'un modèle économique articulé autour d'un service offert gratuitement au consommateur qui est transformé en audience (Smythe, 2001), puis revendu sous sa forme statistique à un public d'annonceurs. Les plateformes comme celles de Google (notamment YouTube et FlickR) répondent à ce fonctionnement. Aucun des trois sites n'utilise de salariés pour produire de contenus originaux. À la place, ils offrent des espaces conviviaux qui mettent en relation producteurs de contenus et consommateurs. Le slogan du site de partage vidéo YouTube, "Broadcast yourself ", est éloquent à ce sujet. En effet, suite à la baisse des coûts d'hébergement (serveurs, disques durs) et de bande passante, YouTube permet aux utilisateurs d'héberger leurs contenus sur ses propres serveurs et de les rendre accessibles au plus grand nombre. La

\footnotetext{
5 "Une des stratégies d'accumulation est de leur donner un libre-accès aux services et aux plateformes, les laisser produire du contenu, et ainsi d'accumuler un grand nombre de producteurs qui sont vendus comme une marchandise aux publicitaires " (traduction libre).
} 


\section{Marc-Olivier GOYETTE-COTE et Philippe LANGLOIS}

popularité incontestable de ce service vidéo (et celle de Facebook et des services offerts par Google également) montre à quel point des services gratuits peuvent réussir à agréger un grand nombre d'utilisateurs en prenant en compte le désir des personnes de s'impliquer dans la chaîne de communication médiatique (Goyette-Côté, 2013).

La source première de revenus pour les plateformes du "web collaboratif " est sans contredit la publicité (Rebillard, 2007 ; Bouquillion et Matthews, 2010 ; Fuchs, 2010). Par conséquent, les efforts pour « rentabiliser» les internautes sont cruciaux pour la réussite économique des plateformes de contenus générés par les utilisateurs. La force de YouTube réside justement dans la valorisation de son audience et la quasi absence de coûts de production. En effet, les goûts des internautes sont comptabilisés en fonction des vidéos consultées, des recherches effectuées et de l'historique de navigation (cookies): ce faisant, le site peut offrir des tranches de publics à ses annonceurs. Pour Facebook, les clients publicitaires peuvent cibler des groupes d'individus en fonction de différents critères, localisations, niveaux d'études, profondeur du lien social des contacts, etc. Ces stratégies de placement publicitaire permettent aux annonceurs de cibler directement une catégorie d'utilisateurs et de ne payer que pour l'exposition de leurs publicités vers des cibles spécifiques. Comme le souligne Fuchs, "google users are double objects of commodification : 1) they and their data are Internet prosumer commodities themselves, 2) through the commodification their consciousness becomes, while online, permanently exposed to commodity logic in the form of advertisement " (2012, p. 44).

II en découle que les prosumers en participant au web dit collaboratif se transforment en marchandises ou, pour reprendre la terminologie de Dallas Smythe, en "audience commodity " (2001). Par ailleurs, " they produce and coproduce parts of the products, and therefore parts of the use value, exchange value, and surplus value that are objectified in these products " ${ }^{6}$ (Fuchs, 2010, p. 191). C'est donc dire que les participants sur les sites Internet «participatifs » subissent une double exploitation: une première provenant de l'exploitation commerciale de leur production et une seconde provenant de leur objectivation en audience revendue à des annonceurs. Derrière le masque de l'émancipation et de la créativité, cette double marchandisation place en réalité le prosumer dans un état de double aliénation, au sens où son double rôle de producteur et de consommateur sur Internet est médiatisé par l'accumulation de capital.

\footnotetext{
${ }^{6}$ "Ils produisent et coproduisent des parties du produit, et donc une partie de la valeur d'usage, de la valeur d'échange et de la plus-value qui sont objectifiées dans ces produits " (traduction libre).
} 
La participation sur les espaces de contenus générés par les utilisateurs, une exploitation collaborative

Fuchs (2010) parle même d'une surexploitation, voire d'une exploitation infinie, puisqu'aucune contrepartie monétaire, rémunération ou salaire n'est donnée aux participants. La "récompense " se limite à l'impression d'avoir contribué au dialogue médiatique et l'espoir d'être reconnu par les autres pour ses interventions sur un site Internet. Selon la théorie de la valeur de Marx, le travail étant la source fondamentale de la valeur, un travail gratuit équivaut à un coefficient d'exploitation infinie. Fuchs mobilise d'ailleurs les équations de la théorie de la valeur pour démontrer ce phénomène. Pour lui, " this shows that produsage in a capitalist society can be interpreted as the outsourcing of productive labor to users who work completly for free and help maximizing the rate of exploitation so that profits can be raised and new media capital may be accumulated ${ }^{7}$ (2010, p. 191).

Nous avons vu, en nous penchant sur la notion de prosumer, que les activités de consommation et de production sont intimement liées (Toffler, 1981; Ritzer et Jurgensson, 2010). Fuchs (2012) va plus loin. Selon l'auteur, les êtres humains sont appelés à produire à tout moment au cours d'une journée, en prenant l'autobus, en faisant l'épicerie, en cuisinant, en écoutant la télévision, etc., ce qui nous amène à nous interroger sur la supposée opposition entre temps de loisir et travail salarié. Le fait que la majorité de la main-d'œuvre en Occident ne travaille plus dans des secteurs industriels classiques fait en sorte qu'il est de plus en plus difficile de séparer temps de travail et temps de loisir. Le travailleur autonome exemplifie bien la difficulté de séparer le temps de travail du reste de la journée de manière précise. En effet, le travailleur autonome peut gérer ses heures de travail et sa productivité, mais plusieurs de ses actions, bien que non rémunérées, sont nécessaires au maintien de son travail (acquisition de connaissances, formation, ateliers, achat de matériel, etc.). Ce qui rejoint les chercheurs marxistes autonomistes italiens Hardt et Negri qui écrivaient dans leur livre Empire, " as labor moves outside of the factory walls, it is increasingly difficult to maintain the fiction of any measure of the working day and thus separate the time of production from the time of reproduction, or work time from leisure time $"^{8}(2000$, p. 403).

\footnotetext{
7 "Cela montre que le "produsage" dans un société capitaliste peut être interprété comme la délocalisation du travail productif vers des utilisateurs qui travaillent gratuitement et aident à maximiser le taux d'exploitation afin que les profits puissent être gonflés et qu'un nouveau capitalmédia puisse être accumulé » (traduction libre).

8 " Au fur et à mesure que le travail se déplace hors des murs de l'usine, il est de plus en plus difficile de maintenir la fiction d'un type de mesure du jour de travail et ainsi séparer le temps de production du temps de reproduction, ou encore le temps de travail du temps de loisir » (traduction libre).
} 


\section{Marc-Olivier GOYETTE-COTE et Philippe LANGLOIS}

Puisqu'il est de plus en plus difficile de différencier les producteurs et les consommateurs, le temps de travail et le temps de loisir, il faut ainsi repenser la notion même de travailleur. Cet éclatement du temps de travail et le développement du travail autonome sont caractéristiques des mouvements de capitaux impliqués dans l'idée d'outsourcing (délocalisation) (Fuchs, 2012). Dans le système néolibéral où une grande importance est attribuée à la rentabilité financière, les coûts variables peuvent être amortis en délocalisant le travail en dehors des murs de l'entreprise, ce qui réduit les dépenses pour les conditions de travail (assurances, pension, échelle salariale, etc.) et en technologie (les coûts étant assumés par les travailleurs) (Fuchs, 2010). Selon Fuchs, la société actuelle dans laquelle le savoir est devenu une marchandise très prisée a donné lieu à une nouvelle classe de travailleurs (knowledge workers) dans le cadre du capitalisme avancé. Ceux-ci contribuent à l'accumulation du capital, soit directement en étant des salariés autonomes, soit indirectement, dans une entreprise, en œuvrant dans la production de biens informationnels destinés à être échangés sur le marché. Avec le développement plus accéléré du secteur économique du savoir et son élargissement à de nouveaux modes de valorisation, nous assistons à une redéfinition des liens d'exploitation du capital.

Le travail gratuit n'est pas un phénomène nouveau propre à l'économie qualifiée d'informationnelle. II s'agit plutôt d'un des aspects essentiels du système capitaliste, un aspect régulièrement mis de côté dans les analyses économiques conventionnelles qui s'attardent uniquement au travail salarié. Ainsi, de nombreuses analyses féministes se sont déjà penchées sur le cas du travail de la mère-épouse-ménagère (Goyette, 1983; Daune-Richard et Devreux, 1992 ; Kergoat, 2000) dont le travail n'est pas rémunéré bien qu'il soit essentiel à la reproduction du système capitaliste (reproduction de la force de travail). Fuchs (2010) va plus loin en avançant que de nombreux autres groupes sociaux produisent du travail - étudiants, aînés, prosumers, bénévoles, etc. qui est approprié gratuitement par le capitalisme. II s'appuie sur les théories de Hardt et Negri qui proposent le concept d'espaces communs dans la société (éducation, sports, culture, etc.) qui, bien qu'ils ne soient pas directement insérés dans l'économie capitaliste, n'en demeurent pas moins tout à fait essentiels à la survie de la société capitaliste. Si ces activités devaient être rémunérées à leur juste valeur, souligne Fuchs, cela entamerait sérieusement la marge de profit globale des entreprises capitalistes. Le prosumer représenterait donc une nouvelle forme de travail gratuit approprié par les capitalistes. Fuchs conclut que l'exploitation des espaces communs effectue une scission dans la société entre ceux qui ne peuvent que participer, par leur travail, à la reproduction de ces espaces communs et ceux qui les exploitent. Les exploités sont qualifiés de multitude, concept de Hardt et Negri qui représente pour Fuchs 
La participation sur les espaces de contenus générés

par les utilisateurs, une exploitation collaborative

le prolétariat au sein du capitalisme moderne. Les exploiteurs sont, bien entendu, les capitalistes, propriétaires des moyens de production qui permettent de s'approprier et de marchandiser le travail, gratuit ou salarié, de la multitude. II est important de noter que dans le capitalisme avancé, cette distinction entre multitude exploitée et capitalistes exploiteurs semble s'embrouiller si l'on se rappelle que par le biais des fonds de pension les travailleurs se retrouvent à jouer un rôle indirect, voire schizophrénique (Aglietta, 1999; Harribey, 2001), de capitalistes.

Cependant, Marx soulignait déjà à son époque que ce sont les rapports sociaux capitalistes qui doivent être considérés comme l'unité de base du système, et non les individus eux-mêmes. Ainsi, un travailleur peut revêtir à la fois le chapeau d'exploité lorsqu'il effectue un travail salarié, et le chapeau d'exploiteur lorsqu'il revient à la maison et s'informe de la rentabilité de ses placements. Tout dépend du type de rapport qu'il entretient avec la société. Cela dit, compte tenu de la faible importance que les revenus financiers jouent chez la majorité des salariés, du très faible contrôle que ceux-ci ont sur leurs investissements et du fait qu'ils sont toujours les premières victimes d'une dégringolade boursière, la distinction classique entre capitalistes possédants et multitude dépossédée tient encore largement la route. Cette distinction permet à Fuchs de renouveler le concept marxiste de classe sociale que l'on qualifie, à tort, d'être trop archaïque pour rendre compte de la complexité des groupes sociaux dans le capitaliste avancé.

\section{La critique de la critique}

Arvidsson et Colleoni (2012), quant à eux, contestent la validité de l'application de la théorie de la valeur de Marx au prosuming. Plus particulièrement, ils contestent l'idée que l'activité des prosumers soit du travail, au sens marxiste du terme. Tout d'abord, la théorie de la valeur affirme que la valeur d'une marchandise est proportionnelle à une certaine quantité nécessaire de travail. Or, ce lien entre valeur et temps serait absent dans l'économie des plateformes de contenus générés par les utilisateurs, car il n'y aurait aucune possibilité de mesurer les pratiques productives pour en faire émerger un quantum moyen de travail, soit le travail abstrait. On retrouve ce point de vue chez Hardt et Negri également qui affirment que cette absence de lien entre valeur, travail et temps rend la théorie de la valeur caduque et obsolète. Ensuite, ces auteurs croient que l'exploitation renvoie nécessairement à une certaine forme de coercition. Or, en l'absence de salaire ou de travail forcé, aucune 


\section{Marc-Olivier GOYETTE-COTE et Philippe LANGLOIS}

coercition ne peut être exercée sur les prosumers, qui choisissent de leur plein gré de participer à la production de contenu, ce qui signifie que leur activité ne peut être considérée comme du travail ou de l'exploitation. Bien qu'ils ne reçoivent aucune rémunération monétaire, les prosumers bénéficient de récompenses affectives (comme la reconnaissance sociale, par exemple) qu'ils retirent de leur engagement envers le contenu produit (Proulx et al, 2011). C'est donc dans cet engagement, sorte "d'entreprenariat de soi », que se trouverait la source de création de la valeur selon Arvidsson et Colleoni. Finalement, ces derniers avancent que les profits perçus par les compagnies qui administrent les plateformes de diffusion de contenu proviennent beaucoup plus de la valorisation financière que des revenus publicitaires. En effet, la majeure partie des profits provient non pas des revenus d'entreprise mais de l'émission et de la vente d'actions, dont le prix est dopé par la spéculation boursière. Pour eux, cela signifie que la réalisation de la valeur ne passe pas par le circuit que dessine la théorie de la valeur marxiste qui prétend que l'on dégage des profits par la vente d'une marchandise, ce qui la rend incapable d'expliquer l'économie informationnelle où la valeur se réalise dans l'espace financier.

Assumant que le prosuming ne peut être considéré comme du travail producteur de valeur appropriée par l'exploitation capitaliste, Arvidsson et Colleoni proposent un modèle alternatif afin de surmonter les insuffisances de la théorie de la valeur marxiste. Selon eux, les compagnies propriétaires des plateformes web de diffusion de contenu cherchent à attirer l'engagement affectif de la multitude en développant une forte image de marque (brand). Cet engagement est alors mesuré par différents outils qui servent à soutenir des conventions financières (Orléans, 1999) afin d'attirer les investisseurs et faire ainsi croître la valeur financière de ces compagnies. Pour eux, le prosuming transforme donc l'engagement affectif en valeur distribuée globalement sur les marchés financiers. Ils donnent l'exemple de Facebook, qui mesure l'engagement par le nombre de membres, de clics, de j'aime, etc., et qui peut, en vertu de sa très grande popularité mesurée pas ces indicateurs, bénéficier d'une capitalisation boursière exceptionnellement grande comparée à sa capacité à générer des revenus, notamment publicitaires. La critique d'Arvidsson et Colleoni envers Fuchs a le mérite de mettre en évidence certaines insuffisances de ce dernier. Selon nous, Fuchs adopte une version trop orthodoxe de la théorie de la valeur dont l'application semble parfois forcée dans le cadre du prosuming. De plus, il emprunte le concept de multitude à des auteurs, Hardt et Negri, qui ont sévèrement critiqué la théorie de la valeur marxiste et la considère, comme on l'a vu précédemment, obsolète dans le cadre du capitalisme informationnel. 
La participation sur les espaces de contenus générés

par les utilisateurs, une exploitation collaborative

Toutefois, l'analyse d'Arvidsson et Colleoni recèle également de multiples lacunes. Tout d'abord, les auteurs entretiennent une maladroite confusion conceptuelle dans leur interprétation de la théorie marxiste. Ils n'établissent aucune distinction entre les termes de valeur, prix, valeur financière, plus-value, valeur-travail, travail abstrait, travail social, etc. qu'ils regroupent tous sous le concept fourre-tout de «valeur ». Les deux auteurs font également preuve d'une conception restreinte du travail qui, pour eux, semble se limiter au salariat. Or, il existe de nombreuses autres formes de travail qui précèdent ou côtoient le travail salarié et qui demeurent invisibles si l'on se limite à une vision conventionnelle comme celle adoptée par ces deux auteurs. D'ailleurs, Fuchs aborde ces différentes formes de travail lorsqu'il discute de la création d'espaces communs. Finalement, si le modèle de l'engagement affectif que développent les deux auteurs présente certains traits intéressants - notamment l'accent mis sur la valorisation financière - il ne semble s'appliquer plus ou moins convenablement qu'à une seule plateforme spécifique : Facebook. Un site comme YouTube, par exemple, correspond beaucoup plus à l'analyse marxiste qu'en donne Fuchs qu'au modèle théorique proposé par Arvidsson et Colleoni.

\section{Vers une nouvelle forme de création de la valeur}

Revenons maintenant à notre point de départ et posons-nous la question suivante: le prosuming est-il du travail ? Est-ce du travail exploité ? Tout dépend en fait des définitions que nous donnons aux termes travail et exploitation. Lorsque le prosumer crée des vidéos qu'il diffuse ensuite sur un site comme YouTube, il y engage sa créativité, son temps, ses propres outils de travail (matériel informatique et logiciels), ses habiletés, son savoir et, bien entendu, son affectivité. Tous ces facteurs représentent un bagage que le prosumer a longuement développé et mûri grâce aux espaces communs de la société comme le système d'éducation. Ses habiletés dépendent donc dans une certaine mesure du travail d'autrui. À notre avis, son activité correspond non seulement à du travail, mais plus encore à du travail social, en ce sens qu'elle produit un contenu qui est destiné à être consommé par l'ensemble de la société et non simplement par le prosumer lui-même.

Ce n'est certes pas du travail salarié, puisqu'aucun salaire n'est versé en échange de cette activité. Est-ce à dire que la mère-épouse-ménagère ne travaille pas parce qu'elle ne reçoit aucun salaire ? Les différents voisins d'un quartier qui se rassemblent pour nettoyer leurs rues ne sont-ils pas en train d'effectuer un travail social bien qu'ils ne reçoivent aucune rémunération 


\section{Marc-Olivier GOYETTE-COTE et Philippe LANGLOIS}

monétaire ? Et pourquoi ces activités deviendraient-elles soudainement du travail si elles sont réalisées par des travailleurs salariés ? Le travail n'est pas une chose en soi, mais un rapport social. Établir des limites strictes autour du concept de travail et d'exploitation en s'arrêtant au travail salarié révèle une conception a-historique du travail qui ne reconnaît, ni la spécificité de la forme proprement capitaliste du travail qu'est le salariat, ni l'existence d'autres formes de travail qui coexistent au sein du système capitaliste et lui sont essentiels. En effet, le salariat se distingue d'autres formes de travail social en ce qu'il consiste en l'appropriation de la force de travail (temps de productivité) des individus par une organisation capitaliste en échange d'une rémunération monétaire. Ce processus n'est pas aussi simple qu'il ne le semble à priori. II suppose d'abord l'existence, ainsi qu'Arvidsson et Colleoni le font remarquer, d'un lien intime entre les notions de travail, de temps et de valeur sous la forme d'un quantum temporel, l'heure de travail rémunérée. Celle-ci représente un développement majeur du système capitaliste qui s'est effectué sur une longue période de temps pour ensuite devenir hégémonique au cours de la révolution industrielle (Diemer et Guillemin, 2009). Elle suppose un haut niveau d'abstraction sociale, une conception radicalement différente du temps par rapport à la notion précapitaliste et, finalement, elle implique l'absorption du temps productif des individus par des organisations capitalistes, propriétaires des moyens de production, qui transforment ce temps en valeur abstraite (Postone, 2009).

Lorsque l'on examine le prosuming, on remarque certains traits caractéristiques du travail salarié, principalement l'absorption et la marchandisation de l'activité humaine par une organisation capitaliste qui la transforme en valeur abstraite sous la forme de profits et de valorisation financière. Ce processus doit servir selon nous de point pivot pour l'analyse du prosuming. Pourtant, ce n'est pas du travail salarié, car il n'y a pas de salaire. Cet aspect est sous-estimé par Fuchs qui, appliquant les formules plus ou moins conventionnelles de la théorie de la valeur, y voit une exploitation infinie. Bien que nous soyons d'accord avec lui sur le fond, les mécanismes nous apparaissent plus subtils. L'absence de salaire rompt le lien qui existe entre temps, travail et valeur propre au salariat, comme le remarquent à la fois Arvidsson et Colleoni, ainsi que Hardt et Negri. Cela revient à dire que l'heure de travail rémunérée ne s'applique pas au prosuming et donc, que les formules que Fuchs utilise pour en arriver à qualifier le prosuming comme exploitation infinie ont en réalité perdu leur pertinence. Plus important encore, ce qui est absorbé par une organisation capitaliste comme YouTube n'est pas le temps de travail, puisqu'il n'y a aucune rémunération basée sur le temps, mais bien les fruits du travail productif des prosumers. Dans ce sens, YouTube ne rémunère pas les créateurs de contenu médiatique en échange de temps productif, elle s'approprie directement et gratuitement les vidéos créées par la masse des 
La participation sur les espaces de contenus générés

par les utilisateurs, une exploitation collaborative

usagers-créateurs, ainsi que des informations sur les utilisateurs (George, 2011) pour soutenir la vente de marchandises publicitaires. YouTube ne s'approprie donc pas du temps d'activité humaine, mais bien des "objets " médiatiques, une production finie et gratuite, fruit des efforts d'une partie importante de la société.

Ainsi, nous pouvons en conclure que le prosuming n'est certes pas du travail salarié et ne doit pas être analysé dans le cadre de modèles destinés à rendre compte du salariat. Cependant, il est du travail en ce qu'il représente de l'activité humaine créative et productive. II est aussi du travail exploité en ce qu'il est une masse de travail social dont la production est absorbée et marchandisée par une organisation capitaliste qui cherche à dégager un profit. Le prosuming doit être perçu dans le cadre plus vaste, propre au système capitaliste, de la marchandisation tous azimuts de l'activité humaine, ou pour reprendre les propos de Fuchs "The total commodification of human creativity " (Fuchs, 2010). II est dans la nature du processus d'accumulation infinie du capital d'intégrer dans le champ de la valeur toujours plus de rapports sociaux afin de dégager une plus-value toujours plus grande (Pineault, 2003, 2007). La création médiatique (YouTube) et les communications humaines sur internet (Facebook) sont ainsi intégrées à l'intérieur de structures capitalistes qui, bien qu'elles ne soient pas salariales, n'en représentent pas moins une exploitation directe de l'activité humaine et du travail social. Comme le fait remarquer Marx, "What the capitalists make use of here are the benefits of the entire system of the social division of labor ${ }^{9}$ (Marx, 1895, p. 175, cité par Fuchs, 2010, p. 186).

\section{Illustrations : Facebook, Amazon et Innocentive}

Cette logique de récupération du travail des utilisateurs est au cœur des stratégies des entreprises possédant les espaces de contenus générés par les utilisateurs. Nous tenterons maintenant d'illustrer notre propos à l'aide des exemples de Facebook, Amazon et Innocentive.

Si nous prenons le cas de Facebook, le modèle d'affaire de la compagnie est basé sur la mise à disposition d'une plateforme développée à l'interne par des travailleurs salariés à des abonnés qui se chargent d'alimenter la plateforme en contenus. Sans le travail gratuit des utilisateurs, la plateforme serait une coquille vide difficilement valorisable. Or, la compagnie propose la plateforme

\footnotetext{
9 «Ce que les capitalistes instrumentalisent ici sont les bénéfices de l'ensemble du système de division sociale du travail » (traduction libre).
} 


\section{Marc-Olivier GOYETTE-COTE et Philippe LANGLOIS}

gratuitement, dans une logique de loss leader (Balcan et al., 2007), puis récupère les pertes encourues par les coûts fixes en vendant les audiences comme marchandises à des annonceurs (Smythe, 2001). Ainsi, Facebook perçoit plus de $95 \%$ de son chiffre d'affaire en publicité, soit 13,4 milliards en 2012 (Pew Research center, 2013), mais ne paie aucune contrepartie aux créateurs de contenus. II s'agit donc d'un travail, puisque l'activité des utilisateurs, que ce soit de produire des mentions "j'aime ", des commentaires ou des publications personnelles, est le résultat concret d'une action dont la valeur est récupérée par l'entreprise, sans contrepartie autre qu'affective. Par ailleurs, cette exploitation est double dans la mesure où Facebook utilise l'activité des utilisateurs pour augmenter l'effet de club de sa plateforme, en plus de l'utiliser pour valoriser sa plateforme à l'aide de publicités de plus en plus ciblées.

Dans le cas de la plateforme Amazon, le consommateur est invité à participer à la notation des produits en plus d'offrir des informations personnelles qui serviront à faire le profilage des consommateurs. II s'agit, selon Kleeman et al. (2008), d'une des formes d'appel au public les plus répandues. II s'agit de mettre en place un système de rétroaction des consommateurs sur le site afin de voir ce qu'ils ont pensé d'un produit $X$ et les autres produits qu'ils recommanderaient pour ceux qui ont aimé X. Amazon a été l'un des pionniers dans ce domaine, permettant aux utilisateurs de donner une note et de laisser un commentaire sur les produits culturels qu'ils avaient aimés. Ce faisant, chaque fois qu'un produit est consulté, le consommateur a accès à des opinions de personnes ayant déjà consommé ce produit. Ce qui dans le contexte où les biens culturels sont des biens d'expérience et que leur valeur n'est connue qu'après avoir été consommés assure aux acheteurs une certaine sécurité sur la nature des biens qu'ils s'apprêtent à acquérir. Dans ce contexte, les consommateurs jouent trois rôles productifs. D'abord, en effectuant des recommandations sur l'offre de produits disponibles, ils effectuent un travail qui relève traditionnellement de celui des libraires. Ensuite, ils créent de la valeur en permettant à la plateforme d'associer différents titres en fonction des historiques de recherche et ainsi de s'attribuer encore une fois une fonction jouée traditionnellement par les libraires, soit regrouper des livres en thématiques. Finalement, ils effectuent un travail d'ingénieur informatique en permettant, par leur usage, d'améliorer l'efficacité des algorithmes de classement et de recherche de produits.

Notre troisième exemple touche plus directement aux transformations des formes d'organisation du travail en proposant un pacte salarié/employeur aux meilleurs contributeurs d'une plateforme. Un exemple de ce type de participation salariée est le site Internet Innocentive.com mis sur pied par Procter \& Gamble 
La participation sur les espaces de contenus générés

par les utilisateurs, une exploitation collaborative

(Howe, 2006). L'idée est d'offrir ici un espace de rencontres entre entreprises et innovateurs. Le service Innocentive est double. Premièrement, il offre aux compagnies de soumettre leurs problèmes de recherche et développement sur l'internet afin que des chercheurs externes à la firme puissent les résoudre. Ainsi, les innovateurs ont accès à une banque de problèmes classés par catégories, par secteur économique ou par rémunération, qui leur permettent d'avoir accès aux problèmes, aux jeux de données et aux recherches déjà entamées sur la question. Le site se distingue d'autres (notamment Amazon Turk) par le volume des rémunérations offertes aux internautes. En effet, les compagnies fixent le prix qu'ils sont prêts à payer pour obtenir des résultats, allant de $1000 \$$ à $100000 \$$ pour certains problèmes plus complexes. L'idée derrière le projet est que l'entretien d'une équipe permanente de recherche et développement est extrêmement onéreux. Pour une question de rétention des cerveaux, les chercheurs de ces grandes compagnies se voient généralement offrir des conditions de travail compétitives. Bien que Procter \& Gamble compte sur une équipe de 9000 chercheurs permanents, il n'est pas dit qu'ils seront capables de développer des produits qui seront mis en marché. Or, avec un système comme celui d'Innocentive, la compagnie a accès à un bassin de plus de 100000 collaborateurs potentiels qui pourraient éventuellement trouver des solutions pour certaines questions de recherche et développement. Ce bassin de contributeurs a l'avantage d'être bien plus diversifié en termes de formations et de compétences que les équipes de recherche maison. De plus, cette main d'œuvre n'est payée que pour les réussites et donc l'entreprise n'a pas à assumer les coûts des échecs des autres innovateurs, mais a seulement à rémunérer un acte, la solution d'un problème, au lieu de payer le salaire, les locaux, le matériel, l'énergie, etc. Du côté des innovateurs l'avantage est clair, faire de l'argent avec des compétences cognitives ou scientifiques tout en y consacrant un nombre limité d'investissements temporels. Cet exemple permet également de soulever un important paradoxe de la participation en ligne. En effet, pour certains, la participation à un site comme Innocentive permet de gagner sa vie en travaillant de la maison. Néanmoins, il y a un risque important pour les contributeurs, à savoir qu'un seul d'entre eux ne soit payé par la plateforme pour ses résultats. C'est donc dire que si 200 personnes travaillent sur un problème, 199 ne seront peut-être pas payés du tout, ce qui constitue une précarisation du travail contractuel.

Avec ces exemples, nous avons illustré concrètement comment la participation des utilisateurs est intégrée dans une stratégie de récupération de la force de travail des internautes. Bien que les utilisateurs puissent avoir des motivations bien à eux (Proulx et al., 2011) - nous pensons aux récompenses affectives et à la présentation de soi dans une logique de mise en marché de 


\section{Marc-Olivier GOYETTE-COTE et Philippe LANGLOIS}

ses qualités de travailleurs dans le but d'attirer l'attention d'un employeur potentiel - elles nous apparaissent secondaires dans l'économie politique des plateformes de contenus générés par les utilisateurs. Ce positionnement s'explique par le fait que nous estimons qu'in fine, c'est la réussite économique de la plateforme qui est l'enjeu au centre de ces dispositifs de participation.

\section{Vers un continuum des formes du travail}

Les ambigüités qui entourent le statut du prosuming prennent racine dans la complexité qui caractérise le travail au sein du capitalisme avancé (Pineault, 2003). Bien que le travail salarié, tel qu'analysé par Marx, y occupe encore une place centrale, le phénomène du prosuming met en relief l'éclatement du travail social dans sa forme typique, le salariat. Cette tendance s'inscrit dans un mouvement global de liquéfaction des rapports sociaux au sein du capitalisme avancé (Pineault, 2003 et 2007). Ce concept renvoie à la dissolution des figures typiques que Marx a analysées, dissolution qui s'effectue parallèlement à

l'approfondissement des rapports capitalistes en eux-mêmes. Par exemple, au temps de Marx, le capital est représenté par la figure traditionnelle du bourgeois $\mathrm{du} 19^{\mathrm{e}}$ siècle dirigeant d'une main de fer sa manufacture, à la fois propriétaire et directeur de son domaine. De nos jours, cette figure s'est déconstruite et dépersonnalisée et le capital s'est liquéfié à travers les grandes corporations qui sont des entités morales autonomes dont la propriété est divisée et dispersée à travers les marchés financiers et qui sont dirigées par des équipes d'administrateurs professionnels. Ainsi, en s'approfondissant, le capital devient un pur processus social quasi intangible et fait éclater le nœud que représentait le personnage du bourgeois. Ce n'est pas un phénomène qui remet en question la pensée de Marx, mais qui au contraire doit être analysé en fonction des rapports capitalistes que celui-ci a mis en évidence, tout en rendant compte de leur flexibilité et de leur nouvelle forme.

Il en va donc de même pour le travail qui maintenant s'exprime sous différentes formes se rapprochant plus ou moins du travail salarié et qui sont plus ou moins directement intégrées et exploités par le système capitaliste. Au sein de ces nouvelles formes, on assiste dans certains domaines comme l'économie informationnelle à la dissolution graduelle de l'heure de travail rémunérée et du salariat comme forme standard du travail social au sein du capitalisme. Ces formes s'inscrivent maintenant dans un continuum du travail où la figure traditionnelle du salariat s'érode peu à peu d'un pôle du continuum à l'autre. Ainsi, de l'ouvrier industriel traditionnel au prosuming et crowdsourcing, en passant par le freelancing, le télé-travail ou le travail autonome, il y a abandon du salaire traditionnel au profit de nouvelles formes de rémunération, 


\section{La participation sur les espaces de contenus générés}

par les utilisateurs, une exploitation collaborative

voire à l'abandon de toute rémunération. Toutes ces activités sont cependant du travail, au sens où elles sont de l'activité humaine créatrice marchandisée, ainsi que nous l'avons déjà souligné. Ce type de travail se différencie de ce que Fuchs appelle le travail créateur d'espaces communs. Alors que le premier est un travail directement exploité en ce qu'il procède immédiatement à une création de valeur pour une compagnie spécifique, le deuxième l'est indirectement en ce qu'il sert à la reproduction globale des structures sociales nécessaires à l'accumulation de capital comme par exemple le travail de la mère-épouse-ménagère. Dans tous les cas, le rapport social est fondamentalement toujours le même: la société est mise au service de l'accumulation du capital dans le cadre de l'extension permanente du champ de la valeur à l'ensemble des rapports qui la constituent.

Est-ce à dire que les TIC ne sont rien d'autre qu'un ensemble d'outils au service de la reproduction du capitalisme, un instrument de domination et d'exploitation ? La présente analyse remet en question l'idée que le web 2.0 est fondamentalement participatif, citoyen, créatif et engagé. Ces qualificatifs peuvent certes, d'un certain point de vue, s'appliquer aux espaces de contenus générés par les utilisateurs, en ce qu'ils rendent compte de l'énorme potentiel d'émancipation sociale que recèle l'internet comme outil de communication, de coopération et de partage. Cependant, ces technologies ont été développées dans le cadre plus global de la société capitaliste et sont insérées à l'intérieur des rapports sociaux proprement capitalistes. Cela veut dire que l'internet, en tant que projet collectif englobant une bonne partie de l'humanité, est subordonné et orienté par l'accumulation de capital. La fourmillante activité de millions d'internautes est chaque jour appropriée, exploitée et transformée en valeur par des organisations capitalistes. II serait pourtant concevable d'envisager l'internet comme un espace social commun qui, comme d'autres espaces communs, devrait échapper aux nécessités du capital. À ce moment seulement pourrait-on considérer que le web deviendrait un bien public favorisant le développement de nouveaux rapports sociaux et non à la poursuite du profit. Ainsi que Marx et Engels l'entrevoyaient dans le Manifeste du parti communiste (1999), le tissu matériel de la société voit son potentiel et son dynamisme freiné par les rapports sociaux de production capitaliste qui l'enchaînent à la nécessité du profit. 


\section{Marc-Olivier GOYETTE-COTE et Philippe LANGLOIS}

\section{Références bibliographiques}

AGLIETTA M., 1999, "Des mutations du capitalisme: une société salariale schizophrène? La revue de la CFDT, $\mathrm{n}^{\circ} 17, \mathrm{pp}$. 14-23.

ARVIDSSON A. et E. COLLEONI, 2012, "Value in Informational Capitalism and on the Internet », The Information Society, vol. 28, n³, pp. 135-150.

BALCAN M.-F., A. BLUM, T-H. H. CHAN $\square$ et M. T. HAJIAGHAVI, 2007, A Theory of Loss-leaders: Making Money by Pricing Below Cost, School of Computer Science, Pittsburgh (PA), Carnegie Mellon University.

BOUQUILLION P. et J. MATTHEWS, 2010, Le Web collaboratif dans le capitalisme: mutations des industries de la culture et de la communication, Grenoble, Presses universitaires de Grenoble.

COMOR E., 2012, «Digital presumption and alienation», Ephemera, vol. 10, $\mathrm{n}^{\circ} 3 / 4$, pp. 439-454.

DAUNE-RICHARD A. et A. DEVREUX, 1992, "Rapports sociaux de sexe et conceptualisation sociologique ", Recherches féministes, vol. 5, n², pp. 7-30.

DIEMER A. et $\mathrm{H}$. GUILLEMIN, 2009, "La marchandisation du travail dans la société anglaise de la fin du XVIle siècle au début du XIXe siècle ", Cahiers d'histoire, n¹10, pp. 52-81.

DUMAZEDIER J., 1962, Vers une civilisation du loisir ?, Paris, Le Seuil.

FUCHS C., 2010, "Labor in Informational Capitalism and on the Internet », The Information Society, $\mathrm{n}^{\circ} 26$, pp. 179-196.

FUCHS C., 2012, « Google capitalism », TripleC - Journal for a Global Sustainable Information Society, vol. 10, $\mathrm{n}^{\circ} 1$, pp.42-48.

GEORGE E. et F. GRANJON, (dir), 2008, Critiques de la société de l'information, Paris, L'Harmattan.

GEORGE E., 2011, "L'intrusion de Google dans la vie privée au cœur des mutations du capitalisme », Terminal, $n^{\circ}$ 108-109, pp. 91-108.

GOYETTE L, 1983, Le travail domestique : entre l'objectivité et la subjectivité : le cas des ménagères au Québec, mémoire de maîtrise, Université Laval.

GOYETTE-CÔTÉ M.-O., 2013, « L'éveil du consommacteur : comment la notion de " prosumer » permet d'analyser les pratiques participatives sur l'internet », dans GEORGE E. et O. KANE (dir.), Actes du colloque Où [en] est la critique en communication, 7 au 11 mai 2012, Montréal, CRICIS, pp. 150-162. 
La participation sur les espaces de contenus générés

par les utilisateurs, une exploitation collaborative

HARDT M. et A. NEGRI, 2000, Empire, Cambridge (Mass), Harvard University Press.

HARRIBEY J.-M., 2001, «La financiarisation du capitalisme et la captation de valeur », dans J.C. DELAUNAY (dir.), Le capitalisme contemporain, Questions de fond, Paris, L'Harmattan, pp. 67-111.

HOWE J., 2006, "The Rise of Crowdsourcing", Wired, vol. 14, ${ }^{\circ} 6$, http://www.wired.com/wired/archive/14.06/crowds pr.html, dernière consultation le 25/05/2014.

KING J.-L., R.E. GRINTER et J.M. PICKERING, 1996, « Grandeur et décadence d'Arpanet: La saga de Netville, cité champignon du cyberespace ", Réseaux, n77, pp. 6-35.

KERGOAT D, 2000, "Division sexuelle du travail et rapports sociaux de sexe » dans HIRATA H., F. LABORIE, H. LE DOARÉ et D. SÉNOTIER, Dictionnaire critique du féminisme, Paris, Presses universitaires de France, pp. 35-44.

KLEEMAN F., G. VOß et K. RIEDER, 2008, "Un(der)paid Innovators: The Commercial Utiliation of Consumer Work through Crowdsourcing ", Science, Technology et Innovation Studies, vol. 4, http://www.stistudies.de/ojs/index.php/sti/article/view/81, dernière consultation le 25/05/2014.

MARX K. et F. ENGELS, 1999, Manifeste du Parti communiste, Paris, Garnier Flammarion. Première édition : 1848.

O'REILLY T., 2005, «What Is Web 2.0 : Design Patterns and Business Models for the Next Generation of Software ", http://oreilly.com/web2/archive/what-is-web20.html, dernière consultation le 25/05/2014.

ORLÉANS A., 1999, Le pouvoir de la finance, Paris, Odile Jacob.

PERRY-BARLOW J., 1996, A Declaration of the Independence of Cyberspace, https://projects.eff.org/ barlow/Declaration-Final.html, dernière consultation le 25/05/2014.

PEW RESEARCH CENTER, 2013, "The Digital Advertising market », dans The state of the News Media, http://stateofthemedia.org/2013/digital-as-mobilegrows-rapidly-the-pressures-on-news-intensify/\#the-digital-advertising-market, dernière consultation le 25/05/2014.

PINEAULT É., 2006, « Au-delà de l'expressionisme et du marxisme : éléments pour une critique des structures sociales du capitalisme avancé ", dans Conférence publique de la chaire MCD tenue à l'UQAM, Montréal, 14 décembre 2006. 


\section{Marc-Olivier GOYETTE-COTE et Philippe LANGLOIS}

PINEAULT É., 2003, Prolégomènes à une critique du capital financier : liquidité du capital et développement de l'institution financière de la modernité, document de travail de la chaire MCD.

POSTONE M., 2009, Temps, travail et domination sociale, Paris, Mille et une nuits.

PROULX S. et A. GOLDENBERG, 2010, «Internet et la culture de la gratuité ", Revue du MAUSS, n³5, pp. 503-517, http://www.cairn.info/revuedu-mauss-2010-1-page-503.htm, dernière consultation le 25/05/2014.

PROULX, S., L. HEATON, L., M.-J. KWOK CHOON et M. MILLETTE, 2011, "Paradoxical empowerment of produsers in the context of informational capitalism », New Review of Hypermedia and Multimedia, vol. 17, n¹, pp. 9-29.

REBILLARD F,. 2007, Le Web 2.0 en perspective : une analyse socio-économique de l'Internet, Paris, L'Harmattan.

RITZER G. et N. JURGENSON, 2010, «Production, Consumption, Prosumption : The Nature of Capitalism in the Age of the Digital "Prosumer" ", Journal of Consumer Culture, vol. 10, n ${ }^{\circ}$, pp. 13-36.

RITZER G., 1983, "The "McDonaldization" of Society ", Journal of American Culture, vol. $6, n^{\circ} 1$, pp. 100-107.

SMYTHE D. W., 2001, "On the Audience Commodity and Its Work», dans DURHAM M. G. et D. M. KELLNER (dir.), Media and Cultural Studies Keyworks, Malden (MA), Blackwell, pp. 230-256.

TOFFLER A., 1981, The Third Wave, Londres, Pan Books Itd. 